\title{
Correction to: DeepGRU: Deep Gesture Recognition Utility
}

\author{
Mehran Maghoumi and Joseph J. LaViola Jr.
}

\section{Correction to:}

Chapter "DeepGRU: Deep Gesture Recognition Utility" in:

G. Bebis et al. (Eds.): Advances in Visual Computing, LNCS 11844, https://doi.org/10.1007/978-3-030-33720-9_2

The given name and family name of an author were not tagged correctly in the originally published article. The author's given name is "Joseph J." and his family name is "LaViola." This was corrected. 